\title{
Geophysical Research Letters
}

\author{
RESEARCH LETTER \\ 10.1029/2019GL086813 \\ Key Points: \\ - Bulk efficiency, which is dominated \\ by rare extreme turbulence events, \\ differs from the corresponding \\ average of patch-wise efficiency \\ - The widely used efficiency of $17 \%$ is \\ supported over a rough bottom \\ where breaking of small-scale \\ internal tides causes enhanced \\ turbulence \\ - Estimated efficiency is as large as \\ $45 \%$ over a sill where enhanced \\ turbulence is thought to be \\ convectively driven by hydraulic \\ overflows
}

Supporting Information:

- Supporting Information S1

Correspondence to:

T. Ijichi,

tijichi@whoi.edu

\section{Citation:}

Ijichi, T., St. Laurent, L., Polzin, K. L., \& Toole, J. M. (2020). How variable is mixing efficiency in the abyss? Geophysical Research Letters, 47, e2019GL086813. https://doi.org/ 10.1029/2019GL086813

Received 23 DEC 2019 Accepted 26 MAR 2020 Accepted article online 28 MAR 2020

(C)2020. American Geophysical Union. All Rights Reserved.

\section{How Variable Is Mixing Efficiency in the Abyss?}

\author{
Takashi Ijichi $^{1}$ (D) Louis St. Laurent ${ }^{2}$ (D) Kurt L. Polzin ${ }^{1}$ (D) and John M. Toole ${ }^{1}$ (D) \\ ${ }^{1}$ Physical Oceanography Department, Woods Hole Oceanographic Institution, Woods Hole, MA, USA, ${ }^{2}$ Applied Physics \\ Laboratory, University of Washington, Seattle, WA, USA
}

\begin{abstract}
Mixing efficiency is an important turbulent flow property in fluid dynamics, whose variability potentially affects the large-scale ocean circulation. However, there are several confusing definitions. Here we compare and contrast patch-wise versus bulk estimates of mixing efficiency in the abyss by revisiting data from previous extensive field surveys in the Brazil Basin. Observed patch-wise efficiency is highly variable over a wide range of turbulence intensity. Bulk efficiency is dominated by rare extreme turbulence events. In the case where enhanced near-bottom turbulence is thought to be driven by breaking of small-scale internal tides, the estimated bulk efficiency is $20 \%$, close to the conventional value of $17 \%$.

On the other hand, where enhanced near-bottom turbulence appears to be convectively driven by hydraulic overflows, bulk efficiency is suggested to be as large as $45 \%$, which has implications for a further significant role of overflow mixing on deep-water mass transformation.
\end{abstract}

Plain Language Summary Ocean turbulence can vertically mix the stratified water column to raise the background gravitational potential energy, maintaining the ocean stratification. "Mixing efficiency" is a parameter representing the fraction of the available turbulent mechanical energy that is irreversibly converted to the background gravitational potential energy during a mixing event. Efficiency has traditionally been treated as a global constant of $17 \%$, but there is growing evidence for its highly variable nature during individual turbulence events. It remains unknown how variable bulk mixing efficiency is (defined as the average over multiple turbulence events, including rare extreme cases). Here we show that bulk efficiency can be significantly different from the corresponding space-time average of local-instantaneous patch-wise efficiency estimates at deep depths with weak stratification. This warns against the application of variable mixing efficiency revealed in individual turbulence events to large-ocean circulation models. The use of the conventional efficiency in such models is supported in a tidal mixing hotspot over rough bathymetry. On the other hand, significantly higher mixing efficiency, up to $45 \%$, is suggested in a localized mixing hotspot over a fracture zone sill, implying a more significant role of overflow mixing on deep-water mass transformation than previously thought.

\section{Introduction}

Ocean turbulence can vertically mix the stratified water column, redistributing heat, dissolved gases, and nutrients, thus greatly affecting the global ocean circulation, marine ecosystems, and ultimately, earth's climate (e.g., Ferrari, 2014; Schmittner, 2005). A better understanding and quantification of turbulent mixing is crucial to improving simulation performance of large-scale ocean circulation models (e.g., MacKinnon et al., 2017). The mixing rate $M$, or the buoyancy flux, is usually quantified in proportion to the turbulent kinetic energy dissipation rate $\varepsilon$, as $M=\Gamma \varepsilon$ with $\Gamma=0.2$, as originally proposed by Osborn (1980). The proportionality coefficient $\Gamma$, or the mixing coefficient, is a measure of mixing efficiency $M /(M+\varepsilon)=\Gamma /(\Gamma+1)$, representing the fraction of the available turbulent mechanical energy that is irreversibly converted to the background gravitational potential energy, which is a key turbulent flow property in stratified fluid dynamics. Despite the wide adoption of the fixed $\Gamma$, many direct numerical simulations, laboratory experiments, and field observations suggest that it is a highly variable statistical quantity during individual turbulence events (e.g., Gregg et al., 2018; Oakey, 1982). Given that the representation of $\Gamma$ is a significant source of uncertainty in large-scale ocean circulation models (de Lavergne et al., 2016; Mashayek, Salehipour et al. 2017; Cimoli et al., 2019), studies on mixing efficiency in the ocean deserve dedicated attention.

One problematic issue is that there are several confusing definitions of mixing efficiency/coefficient in use today, frequently involving different approaches (Gregg et al., 2018). Direct numerical simulations usually focus on individual turbulence events, where the terms "instantaneous" coefficient $\Gamma_{i}(t)=M_{i}(t) / \varepsilon_{i}(t)$ and 
life cycle-averaged "cumulative" coefficient $\Gamma_{c}=\overline{M_{i}(t)} / \overline{\varepsilon_{i}(t)}$ are used (e.g., Peltier \& Caulfield, 2003), where the subscript $i$ denotes an instantaneous value in time $t$ but volume-averaged over the limited numerical domain and the overbar denotes a temporal average over the life span of each turbulence event. From a single microstructure profile, the local-instantaneous or "patch-wise" coefficient $\Gamma_{\text {patch }}(\mathbf{x}, \mathrm{t})=M_{\text {patch }}(\mathbf{x}, \mathrm{t}) /$ $\varepsilon_{\text {patch }}(\mathbf{x}, \mathrm{t})$ may be estimated in terms of the local-instantaneous dissipation rates of thermal variance and turbulent kinetic energy (e.g., Oakey, 1982; see section 2.3 for details), where the subscript "patch" denotes a local-instantaneous value in time $t$ and position $\mathbf{x}$. From many microstructure profiles, on the other hand, the "bulk" coefficient $\Gamma_{\text {bulk }}=M_{\text {patch }}(\mathbf{x}, \mathrm{t}) / \varepsilon_{\text {patch }}(\mathbf{x}, \mathrm{t})$ is estimated in terms of spatiotemporally averaged (or ensemble-averaged) dissipation rates (e.g., St. Laurent \& Schmitt, 1999; see section 2.3 for details), where the angle brackets denote a space-time average over an ensemble large enough to include intermittent rare extreme turbulence events. There is a considerable gap between each of these definitions of $\Gamma$ in space-time scale: the bulk average of the patch-wise coefficient $\Gamma_{\text {patch }}=M_{\text {patch }} / \varepsilon_{\text {patch }}$ is not necessarily equal to the corresponding bulk coefficient $\Gamma_{\text {bulk }}=M_{\text {patch }} / \varepsilon_{\text {patch }}$. It is important to note that a statistically steady state, assumed in the budgets of turbulence kinetic energy (Osborn, 1980) and thermal variance (Osborn \& Cox, 1972), can be expected only in a bulk averaged manner. Given that state-of-the-art large-scale ocean circulation models cannot resolve highly intermittent patchy turbulence events well and therefore must adopt spatiotemporally averaged and statistically steady dissipation and mixing rates within each grid cell, the application of the bulk efficiency is more appropriate for ocean circulation models than the other definitions, in contrast with the recent studies of de Lavergne et al. (2016), Mashayek, Salehipour et al. (2017), and Cimoli et al. (2019) that incorporated characteristics of the instantaneous efficiency into such models.

Here we show how patch-wise versus bulk estimates of mixing efficiency can be different in the abyssal ocean by revisiting data from previous field surveys in the Brazil Basin (western flank of the Mid-Atlantic Ridge), one of the most prominent mixing hotspots in the abyssal interior of the global ocean (Waterhouse et al., 2014). In the pioneering Brazil Basin Tracer Release Experiment (BBTRE), tracer release/microstructure surveys were conducted widely over the Brazil Basin to reveal extraordinarily strong turbulence over the rough bathymetry attributed mainly to breaking of small-scale internal tides (Ledwell et al., 2000; Polzin et al., 1997; St. Laurent et al., 2001). In BBTRE, the bulk $\Gamma$ was suggested to be 0.2, with the general agreement between mixing rates inferred from long-term tracer dispersion and bulk turbulent kinetic energy dissipation estimates taken as primary justification for continuing to use this conventional value in the abyss (Gregg et al., 2018). The more recent Dynamics of Mid-Ocean Ridge Experiment (DoMORE) focused on a specific site in the Brazil Basin, a sill on a canyon floor, across which isopycnals drop precipitously and hydraulic overflow behavior appears dominant (Clément et al., 2017). At this overflow site, intensive microstructure sampling revealed hydraulically driven enhanced near-bottom turbulence. Here the BBTRE and DoMORE microstructure data sets are reexamined to compare and contrast the mixing efficiency characteristics in the tidally driven BBTRE case with those in the hydraulically driven DoMORE case.

\section{Methods}

\subsection{Data}

In BBTRE, the high-resolution profiler (HRP), built at the Woods Hole Oceanographic Institution, was used during cruises in 1996 and 1997 to concurrently measure velocity/temperature/conductivity finestructure and microstructure (Montgomery, 1998; Schmitt et al., 1988). We focused on HRP profiles collected over the eastern Brazil Basin $\left(14^{\circ}-22^{\circ} \mathrm{W}, 19^{\circ}-25^{\circ} \mathrm{S}\right)$, which corresponds to the tracer release/dispersion region (Ledwell et al., 2000). This consists of 33 profiles from the 1996 cruise and 59 profiles from the 1997 cruise. In DoMORE, VMP-6000 vertical microstructure profilers, manufactured by Rockland Scientific International Inc., were used during a cruise in 2015 to concurrently measure velocity/temperature microstructure and temperature/conductivity finestructure (Clément et al., 2017). We used 42 VMP profiles chiefly collected around a major fracture zone sill $\left(14.6^{\circ} \mathrm{W}, 21^{\circ} \mathrm{S}\right)$. Detail profile information is provided in Supporting Information, Figure S1 and Table S1.

\subsection{Turbulent Dissipation Estimates}

Assuming isotropic turbulence, we computed the turbulent kinetic energy dissipation rate $\varepsilon$ and the thermal variance dissipation rate $\chi_{T}$ in 10-m depth segments as $\varepsilon_{10}=7.5 v \overline{u_{z}^{2}}$ and $\chi_{T 10}=6 \kappa \overline{T_{z}^{2}}$, where the subscript 10 
denotes a 10-m segment estimate, $v$ and $\kappa$ are the molecular viscosity and thermal diffusivity of sea water, and $\overline{u_{z}^{2}}$ and $\overline{T_{z}^{2}}$ are the velocity shear variance and the vertical temperature gradient variance. Turbulence variances $\overline{u_{z}^{2}}$ and $\overline{T_{z}^{2}}$ were estimated by spectral analysis (for more details, see Text S1). We computed microstructure spectra averaged over the 10-m depth bins based on half-overlapping 2-s segments of sensor data with Hanning windows applied using instrument-specific algorithms (Douglas \& Lueck, 2016; Polzin \& Montgomery, 1996). At a typical instrument fall rate of about $0.6 \mathrm{~m} \mathrm{~s}^{-1}$, the 2 -s time window corresponds to a depth span of about $1 \mathrm{~m}$. To ensure the quality of our dissipation estimates, we only used simultaneous $\varepsilon$ and $\chi_{T}$ estimates from spectra having reasonable shapes passing a certain criterion of the mean absolute deviation between observed spectra and theoretical spectra in high Reynolds number turbulence (for more details, see Text S2 and Figure S2). Note that we chose the 10-m segment size as a compromise between reducing spectral uncertainty and increasing patch-wise estimates.

\subsection{Patch-Wise and Bulk Estimates of Mixing Coefficient $\Gamma$}

We computed the local-instantaneous or patch-wise mixing coefficient $\Gamma_{\text {patch }}$ every 10-m depth segment of each microstructure profile as

$$
\Gamma_{\text {patch }}=\frac{\chi_{T 10} N_{10}^{2}}{2 \varepsilon_{10} \theta_{z 10}^{2}},
$$

(Oakey, 1982), where $N_{10}^{2}$ and $\theta_{z 10}$ are the background squared buoyancy frequency and potential temperature gradient, respectively, computed by linear fitting of sorted potential density and sorted potential temperature (both referenced to bin-averaged pressure). Subsequently, we computed the spatiotemporally averaged or bulk mixing coefficient $\Gamma_{\text {bulk }}$ in 400-m segments as

$$
\Gamma_{\text {bulk }}=\frac{\left\langle\chi_{T 10}\right\rangle\left\langle N_{10}^{2}\right\rangle}{2\left\langle\varepsilon_{10}\right\rangle\left\langle\theta_{z 10}\right\rangle^{2}},
$$

where the angle brackets denote an average over all available 10-m dissipation estimates from each data set. It is important to note that bulk averaging is essentially required to yield the background "mean" or statistically steady state with wave and eddy components excluded (e.g., Ferrari \& Polzin, 2005; Joyce, 1977). The evaluation of $\Gamma_{\text {patch }}$ could be potentially biased due to inclusions of wave and eddy components in patch-wise stratification. There is some arbitrariness in the domain size for bulk estimates, depending on how many profiles are available. We chose the 400-m vertical size, where 1,848 and 636 individual 10-m dissipation estimates are included on average in the BBTRE and DoMORE cases, respectively, and 95\% bootstrap confidence intervals for $\Gamma_{\text {bulk }}$ are confined within a factor of 2 or less so that $\Gamma_{\text {bulk }}$ exhibits statistically significant depth variations (shown later in section 3.2). If a smaller 200-m vertical size was used, increased statistical uncertainty in the 200-m estimates obscures any significant depth variations (Figure S3).

To reduce possible contamination by salinity-stratified layers and density-compensated intrusions observed at Atlantic mid-depths (Ferrari \& Polzin, 2005; St. Laurent \& Schmitt, 1999), we excluded depth segments with the density stability ratio $R_{\rho}$ ranging $-1 \leq R_{\rho} \leq 2$ from our estimates of $\Gamma_{\text {patch }}$ and $\Gamma_{\text {bulk }}$. Moreover, we excluded an apparent salinity-stratified layer with potential temperature (referenced to 0 dbar) less than $5{ }^{\circ} \mathrm{C}$ and greater than $2.5^{\circ} \mathrm{C}$, which corresponds to a layer between Antarctic Intermediate Water at around 1,000-m depth and North Atlantic Deep Water at around 2,500-m depth (Talley et al., 2011). Note that we focused on $\Gamma_{\text {bulk }}$ only at depth segments deeper than this excluded layer, where enhanced turbulent mixing plays a critical role for an abyssal cell of the Atlantic meridional ocean circulation (e.g., Lumpkin \& Speer, 2007; Nikurashin \& Vallis, 2012; St. Laurent et al., 2001).

\subsection{Relevant Patch-Wise Parameters}

Together with $\Gamma_{\text {patch }}$, the ratio of the Thorpe scale $L_{T}$ to the Ozmidov scale $L_{O}=\left(\varepsilon_{10} / \widehat{N}_{10}^{3}\right)^{1 / 2}$ and the buoyancy Reynolds number $\mathrm{Re}_{\mathrm{b}}=\varepsilon_{10} /\left(\nu_{10} \widehat{N}_{10}^{2}\right)$ were computed, where we estimated the overturn-based buoyancy frequency $\widehat{N}$ and $L_{T}$ from high-resolution potential temperature constructed from microtemperature data to resolve much finer turbulent overturns (for more details, see Ijichi \& Hibiya, 2018). In the BBTRE 
data set, where velocity finestructure data are available, the gradient Richardson number Ri $=\widehat{N}_{10}^{2} / \widehat{S}_{L T}^{2}$ was also computed, where we estimated the background velocity shear for turbulent overturns $\widehat{S}_{L T}$ by linear fitting each observed velocity profile over the computed Thorpe length scale of $L_{T}$. Note that we only computed Ri for $L_{T}>2 \mathrm{~m}$ owing to velocity resolution limitation.

\subsection{Bulk Estimates of Turbulent Diffusivity}

Together with $\Gamma_{\text {bulk }}$, bulk turbulent diffusivities were estimated by the Osborn-Cox model $K_{\mathrm{OC}}$ (Osborn \& Cox, 1972), the Osborn model $K_{\mathrm{O}}$ (Osborn, 1980), and the recent Shih et al. (SKIF) model $K_{\mathrm{SKIF}}$ (Shih et al., 2005), as

$$
\begin{gathered}
K_{\mathrm{OC}}=\left\langle\chi_{T 10}\right\rangle /\left(2\left\langle\theta_{z 10}\right\rangle^{2}\right)=\Gamma_{\text {bulk }}\left\langle\varepsilon_{10}\right\rangle / N_{10}^{2}, \\
K_{\mathrm{O}}=\Gamma_{0} \varepsilon_{10} / N_{10}^{2} \text { and } \\
K_{\mathrm{SKIF}}=\left\{\begin{array}{lll}
\Gamma_{0}\left\langle\varepsilon_{10}\right\rangle / N_{10}^{2} & \cdots & \operatorname{Re}_{\text {bulk }}<\mathrm{Re}_{0} \\
\Gamma_{0}\left(\operatorname{Re}_{\text {bulk }} / \operatorname{Re}_{0}\right)^{-1 / 2} \varepsilon_{10} /\left\langle N_{10}^{2}\right\rangle & \cdots & \operatorname{Re}_{\text {bulk }} \geq \operatorname{Re}_{0}
\end{array},\right.
\end{gathered}
$$

with the bulk buoyancy Reynolds number $\operatorname{Re}_{\text {bulk }}=\varepsilon_{10} /\left(\nu_{10}\left\langle N_{10}^{2}\right\rangle\right), \Gamma_{0}=0.2$, and $\operatorname{Re}_{0}=100$. Note that we did not consider molecular- and buoyancy-controlled regimes with $\mathrm{Re}_{\text {bulk }}<10$ in $K_{\mathrm{SKIF}}$ because much larger values of $\mathrm{Re}_{\text {bulk }}$ were derived for the whole data sets used in this study.

\section{Results}

\subsection{Patch-Wise Estimates}

Wide variations in the patch-wise mixing coefficient $\Gamma_{\text {patch }}$ were observed (Figures 1 and 2). Positive variations of $\Gamma_{\text {patch }}$ with the Thorpe/Ozmidov length scale ratio $L_{T} / L_{O}$ were obtained in both data sets (Figures 1a and 1b), consistent with previous numerical/laboratory/field results (e.g., Rohr \& Van Atta, 1987; Smyth et al., 2001; Mashayek et al. 2017; Ijichi \& Hibiya, 2018). Moreover, in the BBTRE data set, where velocity finestructure data are available, positive variations of $\Gamma_{\text {patch }}$ with the Richardson number Ri are seen (Figure 1c), consistent with previous numerical/laboratory/field results (e.g., Lozovatsky \& Fernando, 2013; Rohr \& Van Atta, 1987). Interestingly, these observed variations are largely consistent with the simple theoretical scalings $\Gamma_{\text {patch }} \propto\left(L_{T} / L_{O}\right)^{4 / 3}$ (Ivey \& Imburger 1991; Ijichi \& Hibiya, 2018) and $\Gamma_{\text {patch }} \propto \mathrm{Ri}$ (Thompson, 1980). It is important to note that these patch-wise estimates span a wide range of turbulence intensities, well above the molecular- and buoyancy-controlled regimes: the buoyancy Reynolds number $\mathrm{Re}_{\mathrm{b}}>>10$ (Figures 2), indicating that they are not affected by differential diffusion (Jackson \& Rehmann, 2014) or anisotropic turbulence (Yamazaki \& Osborn, 1990). Vertical distributions of turbulence intensity in the BBTRE and DoMORE data sets are quite similar such that $\operatorname{Re}_{\mathrm{b}}$ tends to increase with depth. It is apparent from Figure 2 that the recent SKIF (Shih et al., 2005) model of decreasing $\Gamma_{\text {patch }}$ with increasing $\operatorname{Re}_{\mathrm{b}}$ is not supported by either data set: $\Gamma_{\text {patch }}$ actually tends to increase with $\mathrm{Re}_{\mathrm{b}}$ in a moderately energetic regime with $10^{2}<\mathrm{Re}_{\mathrm{b}}<10^{4}$. Moreover, compared with the $L_{T} / L_{O}$ or $\mathrm{Ri}$ space, $\Gamma_{\text {patch }}$ is more broadly distributed over the $\operatorname{Re}_{\mathrm{b}}$ space, suggesting that $\Gamma_{\text {patch }}$ should not be parameterized in terms of $\mathrm{Re}_{\mathrm{b}}$, as argued previously (e.g., Ijichi \& Hibiya, 2018).

In a highly energetic regime with $\mathrm{Re}_{\mathrm{b}} \geq 10^{4}$, where the sample size itself is small but more frequently observed at deeper depths (see histograms in Figure 2), differences in the characteristics of $\Gamma_{\text {patch }}$ between the BBTRE and DoMORE data sets can be seen in spite of large error bars. In the BBTRE case (Figure 2a), $\Gamma_{\text {patch }}$ tends to decrease to the conventional value of 0.2 with increasing $\operatorname{Re}_{\mathrm{b}}$ to $10^{6}$, which is associated with low $L_{T} / L_{O}$ around 1 (Figure 1a) with Ri well below the critical value of 0.25 (Figure 1c) necessary for shear instability development (Miles, 1961). This low-Ri feature supports the scenario that, at the BBTRE site, near-bottom enhanced turbulence is mainly driven by the breaking of small-scale internal tides due to shear instability (Ledwell et al., 2000; Polzin et al., 1997; St. Laurent et al., 2001). It is interesting to note that the observed nonmonotonic dependence of $\Gamma_{\text {patch }}$ on $\mathrm{Re}_{\mathrm{b}}$ is consistent with the recent study of Mashayek, Salehipour et al. (2017). In the DoMORE case (Figure 2b), on the other hand, $\Gamma_{\text {patch }}$ tends to keep high values of around 1 for $\operatorname{Re}_{\mathrm{b}} \geq 10^{4}$, which is associated with high $L_{T} / L_{O}$ around 3 (Figure $1 \mathrm{~b}$ ). It is generally thought 

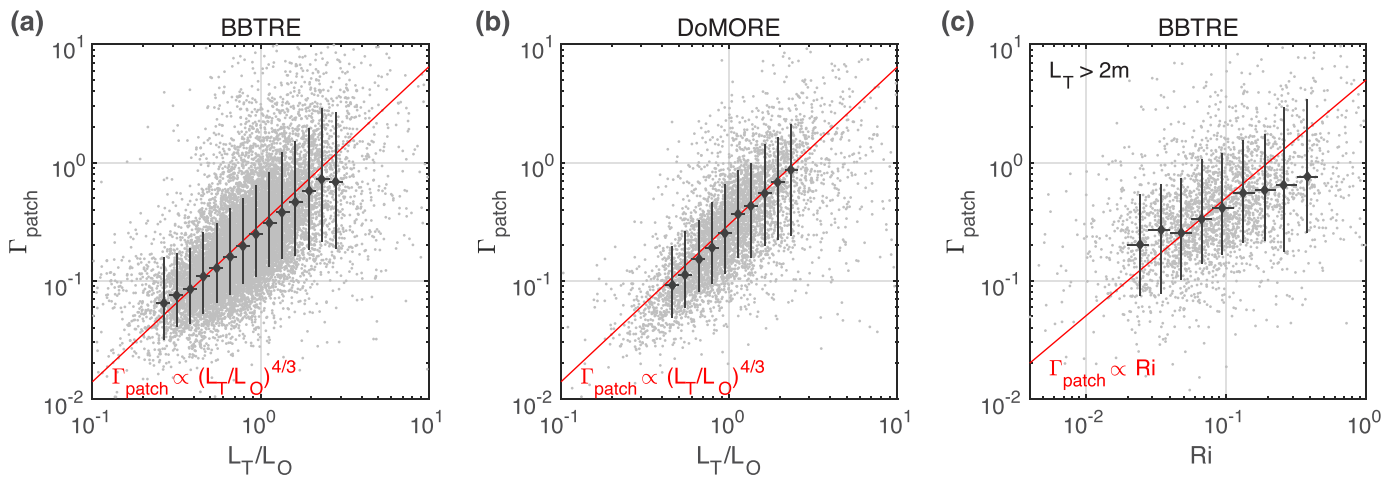

Figure 1. Observed variations of $\Gamma_{\text {patch }}$ with $(\mathrm{a}, \mathrm{b}) L_{T} / L_{O}$ and (c) Ri from (a, c) BBTRE and (b) DoMORE data sets. Black diamonds and vertical bars represent median values and 10th-90th percentile ranges of $\Gamma_{\text {patch }}$ binned by (a, b) $L_{T} / L_{O}$ and (c) Ri as denoted by horizontal bars. Red lines represent the theoretical scalings.

that turbulent flows are more convective (advective) and efficient (higher $\Gamma_{\text {patch }}$ ) for larger $L_{T} / L_{O}$ (Mater et al., 2015; Scotti, 2015). Given that hydraulic overflows are dominant near the bottom at the DoMORE site (Clément et al., 2017), such overflows are suggested to be subject to convective instability, leading to observed strong near-bottom turbulence that mixes the water efficiently. Convective overflow turbulence
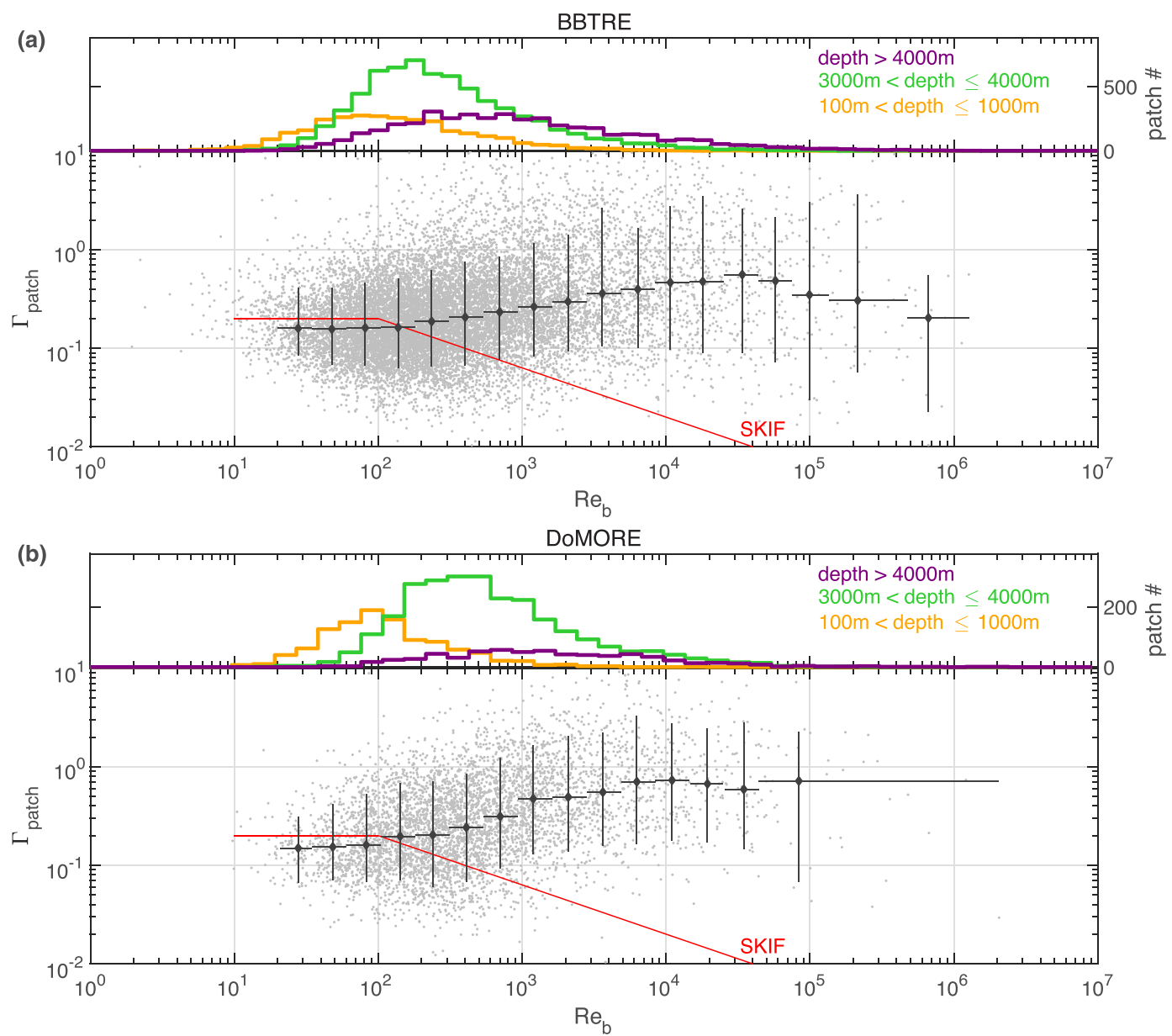

Figure 2. Observed variations of $\Gamma_{\text {patch }}$ with $\mathrm{Re}_{\mathrm{b}}$ from (a) BBTRE and (b) DoMORE data sets. Black diamonds and vertical bars represent median values and 10th-90th percentile ranges of $\Gamma_{\text {patch }}$ binned by $\mathrm{Re}_{\mathrm{b}}$ as denoted by horizontal bars. Red lines represent the Shih et al. (SKIF) model. Histograms above the main scatterplots represent the corresponding probability distribution of $\mathrm{Re}_{\mathrm{b}}$ classified by depth ranges. 

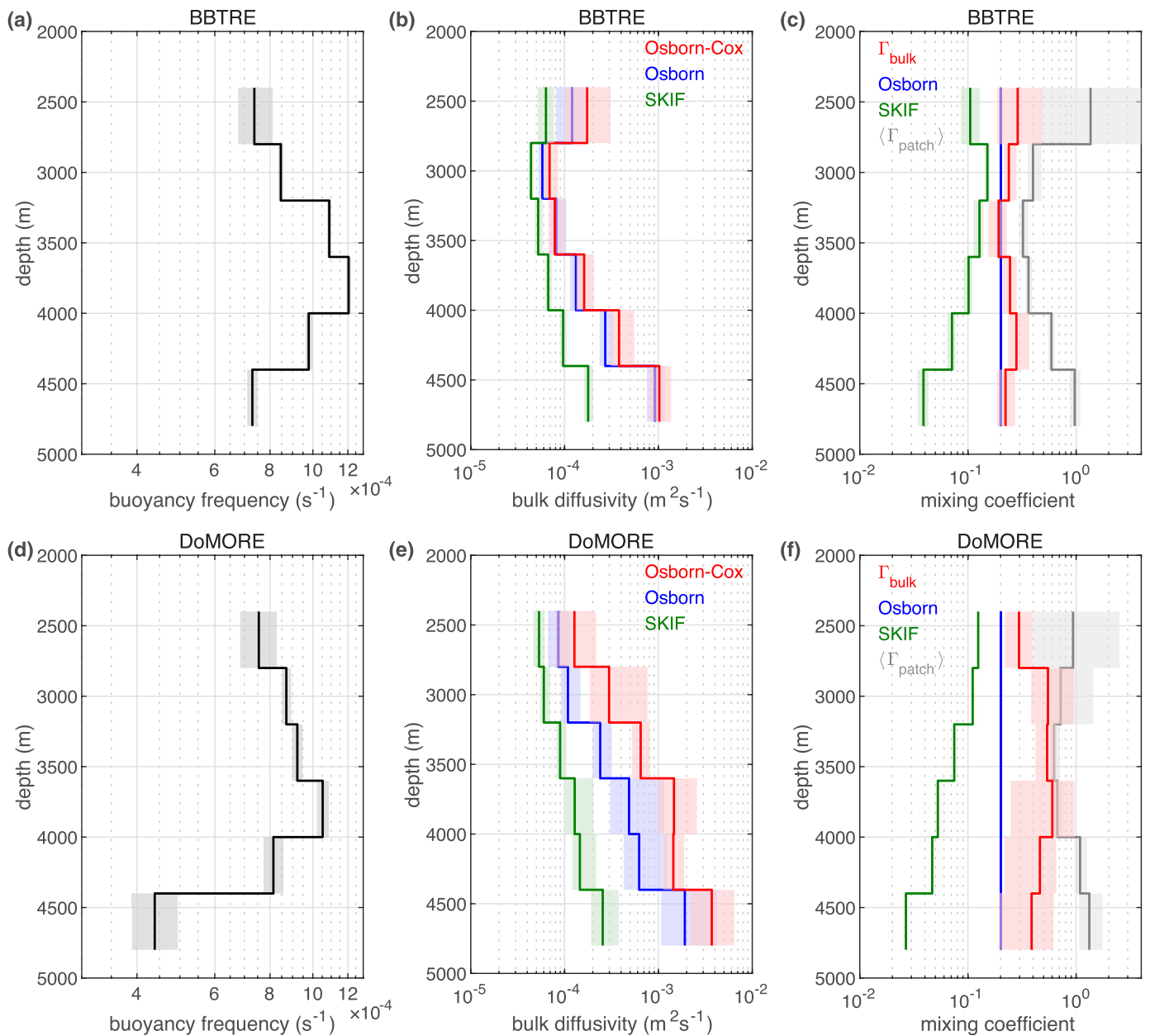

Figure 3. Vertical profiles of (a, d) buoyancy frequency, (b, e) bulk turbulent diffusivities of different models, and (c, f) corresponding mixing coefficients in $(\mathrm{a}-\mathrm{c})$ BBTRE and (d-f) DoMORE cases. Shaded areas represent $95 \%$ bootstrapped confidence intervals.

seems reasonable given that overflow advection of density disturbances could potentially cause a convectively unstable state.

\subsection{Bulk Estimates}

Distinct differences between the BBTRE and DoMORE data sets are also observed in bulk turbulent diffusivities and corresponding mixing coefficients (Figures 3 and 4). In the BBTRE case (Figures 3b, 3c, 4a, and 4b), the bulk diffusivity profile of the Osborn-Cox model (Osborn \& Cox, 1972) is quite similar to that of the Osborn model (Osborn, 1980), with a bulk mixing coefficient $\Gamma_{\text {bulk }}$ of 0.244 , very close to the conventional value of 0.2 , with a $95 \%$ confidence interval ranging between 0.198 and 0.319 . This corresponds to a mixing efficiency of $20 \%$ throughout the deep depth segments. Furthermore, the previous general agreement between the diffusivity inferred from the Osborn model and the tracer dispersion result (Ledwell et al., 2000; Polzin et al., 1997) strongly supports the $\Gamma_{\text {bulk }}$ estimates based on the Osborn-Cox model. In the DoMORE case (Figures 3e, 3f, 4c, and 4d), on the other hand, the bulk diffusivity profile of the Osborn-Cox model is significantly larger than that of the standard Osborn model, with $\Gamma_{\text {bulk }}$ being as large as 0.819 , corresponding to a mixing efficiency of $45 \%$. This is particularly evident in the 3,000- to 4,000-m depth range, in which a major sill is located (see Figure S1). Enhanced $\Gamma_{\text {bulk }}$ over the sill is further apparent in Figure 4d, where $\Gamma_{\text {bulk }}$ is shown to monotonically increase with decreasing height above bottom. It is important to note that these distinct features of near-bottom $\Gamma_{\text {bulk }}$ between the BBTRE and DoMORE data sets are quite consistent with those of $\Gamma_{\text {patch }}$ in the highly energetic regime with $\operatorname{Re}_{\mathrm{b}} \geq 10^{4}$, as shown in section 3.1. However, the data 

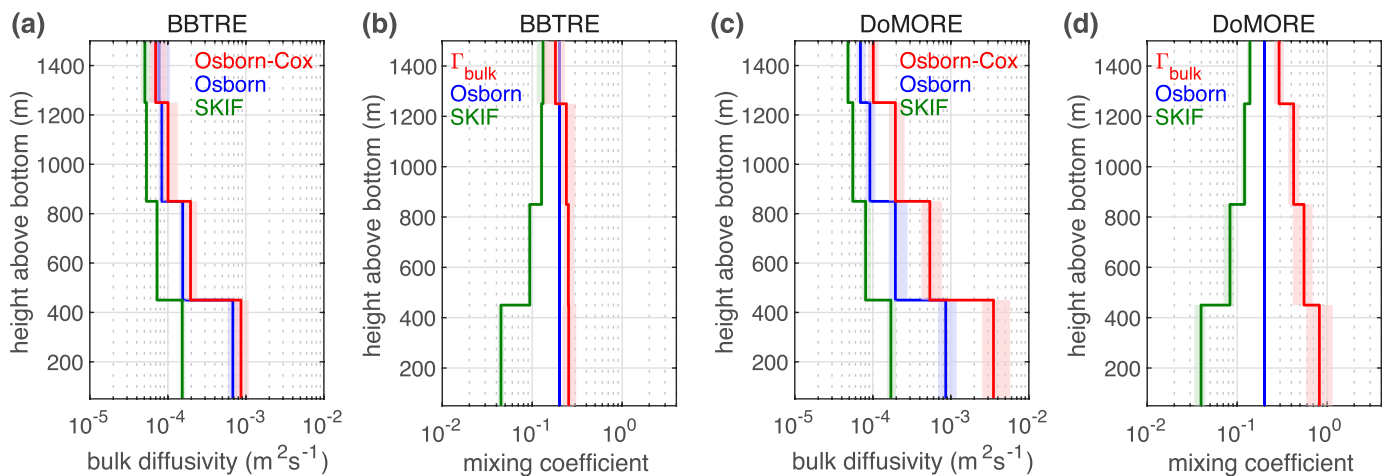

Figure 4. Vertical profiles of (a, c) bulk turbulent diffusivities of different models and (b, d) corresponding mixing coefficients as a function of height above bottom in (a, b) BBTRE and (c, d) DoMORE cases. Shaded areas represent 95\% bootstrapped confidence intervals.

set average of the patch-wise mixing coefficient $\Gamma_{\text {patch }}$ tends to overestimate $\Gamma_{\text {bulk }}$, particularly at deeper depths with weaker stratification (Figures 3a, 3c, 3d, and 3f). This means that, although efficient but moderate turbulence events tend to be more frequently observed at deeper depths, a small number of very energetic but inefficient turbulence events dominate the bulk mixing efficiency, which can be seen as one aspect of turbulence intermittency. If highly energetic turbulent patches with $\operatorname{Re}_{\mathrm{b}} \geq 10^{4}$ are excluded, bulk estimates tend to get larger at deeper depths with weaker stratification in a fashion similar to the corresponding average of patch-wise estimates (see Figure S4). Taking into account that turbulence should be suppressed by the stable background stratification, we speculate that the duration of a turbulence event is so long at weak stratification that a subsequent event is initiated before that first event ceases. Consequently, the young stage of shear-driven turbulence, which is more convective with lower turbulent dissipation rates (e.g., Smyth et al., 2001), might be more frequently sampled at deeper depths with weaker stratification. This is a possible interpretation for the observed larger $\Gamma_{\text {patch }}$ at deeper depths, as previously discussed (Ijichi \& Hibiya, 2018).

Finally, the bulk diffusivity and mixing coefficient of the SKIF model, which was originally made on the basis of characteristics of the instantaneous efficiency (Shih et al., 2005) but was recently applied to a large-scale ocean circulation model (de Lavergne et al., 2016), is assessed through the comparison with that of the Osborn-Cox model (Figures 3 and 4). A decreasing tendency of the mixing coefficient toward the bottom predicted by the SKIF model (Figures 3c, 3f, 4b, and 4d) seems to be compatible with the bottom boundary condition where the buoyancy flux must decrease to zero to satisfy the no-flux condition. As shown in the present study, however, observed $\Gamma_{\text {bulk }}$ never decreases toward the bottom, and the bulk diffusivity of the SKIF model greatly underestimates enhanced near-bottom mixing. We speculate that the mixing coefficient decreases abruptly within several meters of a thin viscous dominated layer to satisfy the bottom boundary condition, which was not captured either in the BBTRE nor the DoMORE data set due to sampling constraints. However, such an abrupt decrease of the mixing coefficient within the thin bottom boundary layer has recently been thought to play an important role in upwelling of cold deep water as part of the global overturning circulation (e.g., Ferrari et al., 2016), observational evidence of which should be explored in the future.

\section{Conclusions}

We have compared and contrasted patch-wise versus bulk estimates of mixing coefficient/efficiency in the abyssal ocean by revisiting data from two previous distinct field surveys in the Brazil Basin, namely, BBTRE and DoMORE. Observed patch-wise efficiency is highly variable over a wide range of turbulence intensity. Bulk efficiency is dominated by a small number of very energetic extreme turbulence events, and the space-time average of patch-wise efficiency tends to overestimate the corresponding bulk efficiency particularly at weak stratification. This warns against the recent studies (de Lavergne et al., 2016; Mashayek, Salehipour et al. 2017; Cimoli et al., 2019) that applied characteristics of the instantaneous efficiency to coarse-resolution ocean circulation models that cannot resolve individual turbulence events. Moreover, the variation of the instantaneous efficiency with the buoyancy Reynolds 
number revealed mostly by direct numerical simulations (Shih et al., 2005) has not been well supported by field observations in the ocean interior except shallow estuarine and coastal regions (e.g., Monismith et al., 2018). As argued by Kunze (2019), this might be owing to numerical difficulties in directly simulating energetic turbulence with large turbulent overturns under weak stratification (i.e., abyssal turbulent flow conditions).

In the BBTRE case, where enhanced near-bottom turbulence is thought to be driven by breaking of small-scale internal tides due to shear instability, the estimated bulk mixing coefficient is 0.24 , with a $95 \%$ confidence interval ranging between 0.20 and 0.32 , and the corresponding efficiency is $20 \%$, further supporting the conventional value of $16.7 \%$, augmenting long-term tracer dispersion results (Gregg et al., 2018). On the other hand, in the DoMORE case, where enhanced near-bottom turbulence is thought to be convectively driven by hydraulic overflows, the bulk mixing coefficient is suggested to be as large as 0.82 and the corresponding efficiency is $45 \%$. Our result of the contrasting values of mixing efficiency related to the different dynamical regimes seems convincing given that they were estimated in the same manner and in similar weakly stratified conditions. Despite being highly localized, strong overflow mixing is thought to greatly affect deep-water mass transformation (e.g., Alford et al., 2013; Polzin et al., 1996). Our finding of larger mixing efficiency than previously thought in overflow sites suggests that overflow mixing may be of even greater importance to the abyssal global ocean. Therefore, it is worth revisiting other overflow mixing hotspots in the future.

\section{Acknowledgments}

TI is a JSPS Overseas Research Fellow. LS, KLP, and JMT acknowledge support from the U.S. National Science Foundation and Office of Naval Research. The authors express their gratitude to Ali Mashayek and an anonymous reviewer for their useful comments on the original manuscript. Data used in this study is available from the Woods Hole Open Access Server (https://hdl.handle.net/1912/25456).

\section{References}

Alford, M. H., Girton, J. B., Voet, G., Carter, G. S., Mickett, J. B., \& Klymak, J. M. (2013). Turbulent mixing and hydraulic control of abyssal water in the Samoan Passage. Geophysical Research Letters, 40, 4668-4674. https://doi.org/10.1002/grl.50684

Cimoli, L., Caulfield, C. P., Johnson, H. L., Marshall, D. P., Mashayek, A., Garabato, A. C. N., \& Vic, C. (2019). Sensitivity of deep ocean mixing to local internal tide breaking and mixing efficiency. Geophysical Research Letters, 46, 14,622-14,633. https://doi.org/10.1029/ 2019GL085056

Clément, L., Thurnherr, A. M., \& Laurent, L. C. S. (2017). Turbulent mixing in a deep fracture zone on the Mid-Atlantic Ridge. Journal of Physical Oceanography, 47, 1873-1896. https://doi.org/10.1175/JPO-D-16-0264.1

Douglas, W., and R. Lueck (2016), ODAS MATLAB Library technical manual, version 4.02, Rockland Scientific International Inc., https:// rocklandscientific.com.

Ferrari, R. (2014). Oceanography: What goes down must come up. Nature, 513(7517), 179-180. https://doi.org/10.1038/513179a

Ferrari, R., Mashayek, A., McDougall, T. J., Nikurashin, M., \& Campin, J.-M. (2016). Turning ocean mixing upside down. Journal of Physical Oceanography, 46, 2239-2261. https://doi.org/10.1175/JPO-D-15-0244.1

Ferrari, R., \& Polzin, K. L. (2005). Finescale structure of the $T-S$ relation in the eastern North Atlantic. Journal of Physical Oceanography, 35, 1437-1454. https://doi.org/10.1175/JPO2763.1

Gregg, M. C., D'Asaro, E. A., Riley, J. J., \& Kunze, E. (2018). Mixing efficiency in the ocean. Annual Review of Marine Science, $10,443-473$. https://doi.org/10.1146/annurev-marine-121916-063643

Ijichi, T., \& Hibiya, T. (2018). Observed variations in turbulent mixing efficiency in the deep ocean. Journal of Physical Oceanography, 48, 1815-1830. https://doi.org/10.1175/JPO-D-17-0275.1

Ivey, G. N., \& Imberger, J. (1991). On the nature of turbulence in a stratified fluid. Part 1: The energetics of mixing. Journal of Physical Oceanography, 21, 650-658. https://doi.org/10.1175/1520-0485(1991)021<0650:OTNOTI >2.0.CO;2

Jackson, P. R., \& Rehmann, C. R. (2014). Experiments on differential scalar mixing in turbulence in a sheared, stratified flow. Journal of Physical Oceanography, 44, 2661-2680. https://doi.org/10.1175/JPO-D-14-0027.1

Joyce, T. M. (1977). A note on the lateral mixing of water masses. Journal of Physical Oceanography, 7, 626-629. https://doi.org/10.1175/ 1520-0485(1977)007<0626:ANOTLM>2.0.CO;2

Kunze, E. (2019). A unified model spectrum for anisotropic stratified and isotropic turbulence in the ocean and atmosphere. Journal of Physical Oceanography, 49, 385-407. https://doi.org/10.1175/JPO-D-18-0092.1

de Lavergne, C., Madec, G., Sommer, J. L., Nurser, A. J. G., \& Naveira Garabato, A. C. (2016). The impact of a variable mixing efficiency on the abyssal overturning. Journal of Physical Oceanography, 46, 663-681. https://doi.org/10.1175/JPO-D-14-0259.1

Ledwell, J. R., Montgomery, E. T., Polzin, K. L., Laurent, L. C. S., Schmitt, R. W., \& Toole, J. M. (2000). Evidence for enhanced mixing over rough topography in the abyssal ocean. Nature, 403(6766), 179-182. https://doi.org/10.1038/35003164

Lozovatsky, I. D., \& Fernando, H. J. S. (2013). Mixing efficiency in natural flows. Philosophical Transactions of the Royal Society A, 371(1982). https://doi.org/10.1098/rsta.2012.0213

Lumpkin, R., \& Speer, K. (2007). Global ocean meridional overturning. Journal of Physical Oceanography, 37, 2550-2562. https://doi.org/ $10.1175 / \mathrm{JPO} 3130.1$

MacKinnon, J. A., Zhao, Z., Whalen, C. B., Waterhouse, A. F., Trossman, D. S., Sun, O. M., et al. (2017). Climate process team on internal wave-driven ocean mixing. Bulletin of the American Meteorological Society, 98(11), 2429-2454. https://doi.org/10.1175/ BAMS-D-16-0030.1

Mashayek, A., Caulfield, C. P., \& Peltier, W. R. (2017). Role of overturns in optical mixing in stratified mixing layers. Journal of Fluid Mechanics, 826, 522-552. https://doi.org/10.1017/jfm.2017.374

Mashayek, A., Salehipour, H., Bouffard, D., Caulfield, C. P., Ferrari, R., Nikurashin, M., et al. (2017). Efficiency of turbulent mixing in the abyssal ocean circulation. Geophysical Research Letters, 44, 6296-6306. https://doi.org/10.1002/2016GL072452

Mater, B. D., Venayagamoorthy, S. K., Laurent, L. S., \& Moum, J. N. (2015). Biases in Thorpe-scale estimates of turbulence dissipation. Part I: Assessments from large-scale overturns in oceanographic data. Journal of Physical Oceanography, 45, 2497-2521. https://doi.org/ 10.1175/JPO-D-14-0128.1 
Miles, J. (1961). On the stability of heterogeneous shear flows. Journal of Fluid Mechanics, 10, 496-508. https://doi.org/10.1017/ S0022112061000305

Monismith, S. G., Koseff, J. R., \& White, B. L. (2018). Mixing efficiency in the presence of stratification: When is it constant? Geophysical Research Letters, 45, 5627-5634. https://doi.org/10.1029/2018GL077229

Montgomery, E. T. (1998. Use of the high resolution profiler (HRP) in the Brazil Basin Tracer Release Experiment, Woods Hole Oceanographic Institution Technical Report, WHOI-98-08, 38 pp.

Nikurashin, M., \& Vallis, G. (2012). A theory of the interhemispheric meridional overturning circulation and associated stratification. Journal of Physical Oceanography, 42, 1652-1667. https://doi.org/10.1175/JPO-D-11-0189.1

Oakey, N. S. (1982). Determination of the rate of dissipation of turbulent energy from simultaneous temperature and velocity shear microstructure measurements. Journal of Physical Oceanography, 12, 256-271. https://doi.org/10.1175/1520-0485(1982)012<0256: DOTROD>2.0.CO;2

Osborn, T. R. (1980). Estimates of the local rate of vertical diffusion from dissipation measurements. Journal of Physical Oceanography, 10, 83-89. https://doi.org/10.1175/1520-0485(1980)010<0083:EOTLRO >2.0.CO;2

Osborn, T. R., \& Cox, C. S. (1972). Oceanic fine structure. Geophysical Fluid Dynamics, 3, 321-345. https://doi.org/10.1080/ 03091927208236085

Peltier, W. R., \& Caulfield, C. P. (2003). Mixing efficiency in stratified shear flows. Annual Review of Fluid Mechanics, 35, 135-167. https:// doi.org/10.1146/annurev.fluid.35.101101.161144

Polzin, K. L., \& Montgomery, E. T. (1996). Microstructure profiling with the high resolution profiler. In Proceedings of the ONR Workshop on Microstructure Sensors (pp. 109-115). Mt. Hood, OR: Office of Naval Research.

Polzin, K. L., Speer, K. G., Toole, J. M., \& Schmitt, R. W. (1996). Intense mixing of Antarctic bottom water in the equatorial Atlantic Ocean. Nature, 380(6569), 54-57. https://doi.org/10.1038/380054a0

Polzin, K. L., Toole, J. M., Ledwell, J. R., \& Schmitt, R. W. (1997). Spatial variability of turbulent mixing in the abyssal ocean. Science, 276, 93-96. https://doi.org/10.1126/science.276.5309.93

Rohr, J., \& Van Atta, C. (1987). Mixing efficiency in stably stratified growing turbulence. Journal of Geophysical Research, 92(C5), 5481-5488. https://doi.org/10.1029/JC092iC05p05481

Schmitt, R. W., Toole, J. M., Koehler, R. L., Mellinger, E. C., \& Doherty, K. W. (1988). The development of a fine- and microstructure profiler. Journal of Atmospheric and Oceanic Technology, 5, 484-500. https://doi.org/10.1175/1520-0426(1988)005<0484: TDOAFA>2.0.CO;2

Schmittner, A. (2005). Decline of the marine ecosystem caused by a reduction in the Atlantic overturning circulation. Nature, 434, 628-633. https://doi.org/10.1038/nature03476

Scotti, A. (2015). Biases in Thorpe-scale estimates of turbulence dissipation. Part II: Energetics arguments and turbulence simulations. Journal of Physical Oceanography, 45, 2522-2543. https://doi.org/10.1175/JPO-D-14-0092.1

Shih, L. H., Koseff, J. R., Ivey, G. N., \& Ferziger, J. H. (2005). Parameterization of turbulent fluxes and scales using homogeneous sheared stably stratified turbulence simulations. Journal of Fluid Mechanics, 525, 193-214. https://doi.org/10.1017/ S0022112004002587

Smyth, W. D., Moum, J. N., \& Caldwell, D. R. (2001). The efficiency of mixing in turbulent patches: Inferences from direct simulations and microstructure observations. Journal of Physical Oceanography, 31, 1969-1992. https://doi.org/10.1175/1520-0485(2001)031<1969: TEOMIT $>2.0 . \mathrm{CO} ; 2$

St. Laurent, L., \& Schmitt, R. W. (1999). The contribution of salt fingers to vertical mixing in the North Atlantic Tracer Release Experiment. Journal of Physical Oceanography, 29, 1404-1424. https://doi.org/10.1175/1520-0485(1999)029<1404:TCOSFT>2.0.CO;2

St. Laurent, L. C., Toole, J. M., \& Schmitt, R. W. (2001). Buoyancy forcing by turbulence above rough topography in the abyssal Brazil basin. Journal of Physical Oceanography, 31, 3476-3495. https://doi.org/10.1175/1520-0485(2001)031<3476:BFBTAR>2.0.CO;2

Talley, L. D., Pickard, G. L., Emery, W. J., \& Swift, J. H. (2011). Descriptive physical oceanography: An introduction (6th edition, p. 560). Burlington, MA: Elsevier, Academic Press.

Thompson, R. O. R. Y. (1980). Efficiency of conversion of kinetic energy and potential energy by breaking internal waves. Journal of Geophysical Research, 85(C11), 6631-6635. https://doi.org/10.1029/JC085iC11p06631

Waterhouse, A. F., MacKinnon, J. A., Nash, J. D., Alford, M. H., Kunze, E., Simmons, H. L., et al. (2014). Global patterns of diapycnal mixing from measurements of the turbulent dissipation rate. Journal of Physical Oceanography, 44(7), 1854-1872. https://doi.org/ 10.1175/JPO-D-13-0104.1

Yamazaki, H., \& Osborn, T. (1990). Dissipation estimates for stratified turbulence. Journal of Geophysical Research, 95(C6), 9739-9744. https://doi.org/10.1029/JC095iC06p09739 